\title{
On some possible extensions of the Brodsky-Lepage-Mackenzie approach beyond the next-to-leading order
}

\author{
G. Grunberg \\ Centre de Physique Theorique, Ecole Polytechnique, F-91128 Palaiseau, France
}

and

\author{
A.L. Kataev ${ }^{1}$ \\ Randall Laboratory of Physics, University of Michigan. Ann Arbor, MI 48109-1120, USA
}

Received 20 May 1991; revised manuscript received 20 January 1992

\begin{abstract}
Noting that the choice of renormalization point advocated by Brodsky, Lepage and Mackenzic (BLM) is the flavor independent prescription which removes all $f$-dependence from the next-to-leading order coefficients, we consider the possible generalization which requires all higher order coefficients $r_{i}$ to be $f$-independent constants $r_{i}^{*}$. We point out that in QCD, setting $r_{i}=r_{i}^{*}$ is always possible, but leaves us with an ambiguous prescription. We consider an alternative possibility within the framework of the BLM approach and apply the corresponding prescription to the next-to-next-to-leading approximation of $\sigma_{\mathrm{tot}}\left(\mathrm{e}^{+} \mathrm{e}^{-} \rightarrow\right.$ hadrons $)$ in QCD. The analogous questions and the special features of the BLM and effective charge approaches in QED are also discussed.
\end{abstract}

1. Fixing the arbitrariness of the renormalization scheme (RS) is of much practical interest (both thcoretically and phenomenologically) in perturbative $\mathrm{QCD}$, owing to the not so small value of the coupling constant. In the next-to-leading order (NLO) one has to fix the scheme renormalization point $\mu$ or the definition of the parameter $A$. In the next-to-next-toleading order (NNLO) one has to fix the scheme-dependent NNLO coefficient of the renormalization group ( $R G) \beta$-function.

Brodsky, Lepage and Mackenzie (BLM) have proposed, for any given physical quantity $R$, an interesting flavor independent prescription to fix $\mu$ (or $A$ ) which removes all flavor $f$-dependence from the NLO coefficient $r_{1}$ of the perturbative series for $R$ [1]. The motivation of this prescription is the observation that in QED the energy dependence of the invariant charge can be identificd with the renormalized expression of

\footnotetext{
1 Present and permanent address: Institute for Nuclear Rcsearch, SU-117312 Moscow, USSR.
}

the photon vacuum polarization graphs. Starting from this observation BLM have proposed to absorb the fermion contributions to the photon (or gluon) propagators to the scale fixing parameter $\mu$ (or $A$ ). Within this approach one can consider the question of applicability (or non-applicability) of the perturbative predictions for their detailed comparison with experimental data [1].

This proposal has already been discussed and used in a number of phenomenological QCD studies (see e.g. ref. [2] ). However, the problem of the possibility of the extension of the BLM proposal beyond the NL level remained open (recently this problem was also investigated within the skeleton expansion in ref. [3] ). In this note we discuss the generalization of the BLM proposal which requires all higher order coefficients $r_{i}$ to be independent from $f$ as well. We will show that beyond the NLO, in addition to the prescription to fix $\mu$, one must consider the problem how to fix the higher order coefficients of the RS $\beta$-function. We will find that this problem has no unique 
solution. We then consider the alternative procedure of using a fixed RS $\beta$-function, but a coupling constant dependent redefinition of $\mu$ in higher orders of perturbation theory [1]. As an example we consider the application of this prescription to deal with the scheme dependence ambiguity of the NNLO QCD approximation of $\sigma_{\mathrm{tot}}\left(\mathrm{e}^{+} \mathrm{e}^{-} \rightarrow\right.$ hadrons $)$. The analogous questions in QED are also discussed.

2. Consider a physical quantity $R=R(Q)$ and its expression in an arbitrary RS with the coupling $a=\alpha_{\mathrm{s}} / \pi, a=a(\mu)$ :

$R=a\left(1+r_{1} a+r_{2} a^{2}+r_{3} a^{3}+\ldots\right)$.

If we restrict ourselves to RS which does not introduce the arbitrary $f$-dependence beyond that expected from fermion-loop insertions, $r_{i}$ will be a linear function of $f$, and since the same is truc for the one-loop coefficient $\beta_{0}$ of the RG QCD $\beta$-function

$$
\begin{aligned}
& \mu^{2} \frac{\partial a}{\partial \mu^{2}}=\beta(a) \\
& \quad=-\beta_{0} a^{2}-\beta_{1} a^{3}-\beta_{2} a^{4}-\beta_{3} a^{5}-\ldots,
\end{aligned}
$$

one can write

$r_{1}=-\beta_{0}\left(\ln \frac{Q^{2}}{\mu^{2}}+d_{1}^{*}\right)+r_{1}^{*}$,

where both $d_{1}^{*}$ and $r_{1}^{*}$ are $f$-independent. BLM propose to start from the $\overline{\mathrm{MS}} \overline{\mathrm{S}}$-scheme [4] and to fix $\mu=\mu_{*}$ by the condition $\ln \left(Q^{2} / \mu_{*}^{2}\right)+d_{1}^{*}=0$, so that $r_{1}=r_{1}^{*}$. Writing $r_{1}=r_{10}+r_{11} f$ and $\beta_{0}=\beta_{00}+\beta_{01} f$ one has

$r_{10}=-\beta_{00}\left(\ln \frac{Q^{2}}{\mu^{2}}+d_{1}^{*}\right)+r_{1}^{*}$

and

$r_{11}=-\beta_{01}\left(\ln \frac{Q^{2}}{\mu^{2}}+d_{1}^{*}\right)$

The BLM proposal is therefore equivalent to the condition $r_{11}^{\overline{M S}}=0$ or $\mu_{*}^{2}=Q^{2} \exp \left(d_{1}^{*}\right)$, i.e., that $r_{1}$ is $f$-independent, with $f, \mu$ and $Q$ naturally considered as independent variables. Note that the BLM pres- ciption can be restated as, first, a redefinition of $\mu: \mu_{\overline{\mathrm{MS}}}^{\frac{2}{2}} \rightarrow \mu_{\mathrm{BLM}}^{2}=\mu_{\mathrm{MS}}^{\frac{2}{2}} \exp \left(-d_{1}^{*}\right)$, or equivalently of $\Lambda: \quad \Lambda_{\mathrm{MS}}^{\frac{2}{\mathrm{MS}}} \rightarrow \Lambda_{\mathrm{BLM}}^{2}=\Lambda_{\overline{\mathrm{MS}}}^{\frac{2}{\mathrm{~S}}} \exp \left(-d_{1}^{*}\right)$, with $d_{1}^{*}=$ $-r_{11}^{\overline{M S}}(\mu=Q) / \beta_{01}$ (this is an analogue to the transition from the MS to the $\overline{\mathrm{MS}}$-scheme [4]). The second step is to put $\mu_{\mathrm{BLM}}^{2}=Q^{2}$. Note, that since $r_{11}^{\overline{M S}}$ is process dependent, the definition of $\mu_{\mathrm{BLM}}^{2}$ is process dependent too.

A straightforward extension of the BLM scheme then consists in requiring all $r_{i}$ to be also $f$-independent. We first show that it is always possible to achieve this for any given $R$, by choosing arbitrary, f-independent values for the $r_{i}$. To see this it is convenient to use the RS-invariant effective charge approach [58 ] where the RS coupling $a_{\mathrm{eff}}$ is identified with $R$ itself, $R=a_{\mathrm{eff}}$ (see also ref. [9]). The corresponding RG equation for $R$ then defines the effective charge $\beta$-function:

$$
\begin{aligned}
& Q^{2} \frac{\mathrm{d} R}{\mathrm{~d} Q^{2}}=\beta_{\mathrm{eir}}(R) \\
& \quad=-\beta_{0} R^{2}-\beta_{1} R^{3}-\tilde{\beta}_{2} R^{4}-\tilde{\beta}_{3} R^{5}-\ldots,
\end{aligned}
$$

which is the RS-invariant object which governs the $Q^{2}$-evolution of $R$ in QCD. In particular, the $\tilde{\beta}_{i}(i \geqslant$ 2 ) are the RS-invariant quantitics [5-7] (simply related to the scheme-invariants $\rho_{t}, i \geqslant 2$, introduced in ref. [10]). Upon solving eq. (6) one introduces the effective RS-invariant scale parameter $A_{\text {eff, }}$ such that

$\beta_{0} \ln \frac{Q^{2}}{\Lambda_{\mathrm{eff}}^{2}}=\frac{1}{R}+\mathrm{O}(\ln R)+$ const. $+\mathrm{O}(R)$

$\left(\beta_{0} \ln \left(Q^{2} / \Lambda_{\mathrm{cff}}^{2}\right)\right.$ is connected with the invariant $\rho_{1}$ of ref. [10]). On the other hand, the solution of the RG equation (3), with $a\left(\mu^{2}\right)$ defined in the considered RS, reads

$\beta_{0} \ln \frac{\mu^{2}}{A^{2}}=\frac{1}{a}+\mathrm{O}(\ln a)+$ const. $+\mathrm{O}(a)$,

and introduces the RS scale parameter $\Lambda$. The effective charge RS-invariant parameters $\Lambda_{\text {eff }}$ and $\widetilde{\beta}_{i}$ are related to the RS parameter $\Lambda$ and $\beta_{i}$ by the relations $[5-7,11]$

$r_{1}-\beta_{0} \ln \frac{\mu^{2}}{\Lambda^{2}}=-\beta_{0} \ln \frac{Q^{2}}{\Lambda_{\mathrm{eff}}^{2}}$,
$\beta_{0}\left(r_{2}-r_{\mathrm{i}}^{2}\right)=\beta_{1} r_{1}+\widetilde{\beta}_{2}-\beta_{2}$, 


$$
\begin{aligned}
& \beta_{0}\left(r_{3}-r_{1}^{3}\right) \\
& \quad=\frac{5}{2} \beta_{1} r_{1}^{2}+\left(3 \tilde{\beta_{2}}-2 \beta_{2}\right) r_{1}+\frac{1}{2}\left(\tilde{\beta_{3}}-\beta_{3}\right),
\end{aligned}
$$

etc. Eq. (9) follows immediately by taking the difference between eq. (7) and eq. (8), reexpanding in powers of $a$ and comparing with the standard expansion of eq. (1). It is clear from eqs. (10) and (11) that one can assign arbitrary values to $r_{1}, r_{2}, r_{3}, \ldots$ at $\mu=Q$ which will determine both $A$ [by $\beta_{0} \ln \left(A^{2} /\right.$ $\left.\left.A_{\text {eff }}^{2}\right)=-r_{1}(\mu=Q)\right]$ and $\beta_{2}, \beta_{3}, \ldots$, i.e., the renormalization scheme parameters (this fixes the schemc). In particular, one can take the $r$, to be $f$-independent constants. This conclusion can be generalized to all orders using the relation $\beta(a)(\partial R / \partial a)=\beta_{\text {efr }}(R)$ which determines the $\beta_{i}$ given the $r_{i}$ and the $\tilde{\beta},[6,7,11]$ (sec ref. [12] for explicit formulac).

We next investigate what the constraints are on the RS $\beta$-functions which follow from the requirement that the $r_{i}$ are $f$-independent. We first show that this condition cannot be achieved by the mere BLM choice of $\mu$, but imply additional restrictions on the RS dependent $\beta$-function coefficients. We shall only consider the schemes where the general form of $f$-dependence is the same as expected if induced solely by fermion-loop insertions, namely that $r_{i}$ are polynomial in $f$ of maximal degree $i$. Thus we assume

$r_{1}=r_{10}+r_{11} f, \quad r_{2}=r_{20}+r_{21} f+r_{22} f^{2}$,

$r_{3}=r_{30}+r_{31} f+r_{32} f^{2}+r_{33} f^{3}$,

etc., and also

$\beta_{0}=\beta_{00}+\beta_{01} f, \quad \beta_{1}=\beta_{10}+\beta_{11} f$,

$\beta_{2}=\beta_{20}+\beta_{21} f+\beta_{22} f^{2}+\beta_{23} f^{3}$,

$\tilde{\beta}_{2}=\tilde{\beta}_{20}+\widetilde{\beta}_{21} f+\tilde{\beta}_{22} f^{2}+\widetilde{\beta}_{23} f^{3}$.

Note that the $\widetilde{\beta}_{2}$-coefficient can have $f^{3}$-terms as has been observed [13] to occur on the basis of the recent NNLO O $\left(a^{3}\right)$ calculations [14,13,15] (the QCD results [15] are in agreement with the ones presented in ref. [16], for a critical discussion see ref. [17]), although they are absent in the MS-scheme [18] and MOM scheme [19]. We generalize the foundations of ref. [13] and stress that once the $f^{3}$-terms appeared in the $\widetilde{\beta}_{2}$-coefficients in the effective scheme, they can also exist in another arbitrary scheme, and that in general $\beta_{23} \neq 0$.

Note also that some special contributions to the $f$ - dependence like light-by-light scattering diagrams in QED, which form a class of RG-invariant diagrams, may and probably should be treated separately.

Let us now consider the condition for $r_{2}$ to be $f-$ independent. Inserting eqs. (12) and (13) into eq. (10) and taking $r_{1}=r_{1}^{*}=r_{10}^{*} f$-independent, one gets a set of relations which determine the corresponding coefficient $r_{2}=r_{20}+r_{21} f+r_{22} f^{2}$ at $\mu=\mu_{*}$, namely $\bar{r}_{2}=$ $\bar{r}_{20}+\bar{r}_{21} f+\bar{r}_{22} f^{2}$.

$\bar{r}_{22}=\frac{\tilde{\beta}_{23}-\beta_{23}}{\beta_{01}}$,

$\tilde{r}_{21}=\frac{\tilde{\beta}_{22}-\beta_{22}}{\beta_{01}}-\frac{\beta_{00}}{\beta_{01}^{2}}\left(\tilde{\beta}_{23}-\beta_{23}\right)$,

$\bar{r}_{20}-r_{1}^{* 2}=\frac{\beta_{11}}{\beta_{01}} r_{1}^{*}-\frac{\beta_{00}}{\beta_{01}} \bar{r}_{21}+\frac{\tilde{\beta}_{21}-\beta_{21}}{\beta_{01}}$,

$\bar{r}_{20}-r_{1}^{* 2}=\frac{\beta_{10}}{\beta_{00}} r_{1}^{*}+\frac{\widetilde{\beta}_{20}-\beta_{20}}{\beta_{00}}$.

Eqs. (16) and (17) allow to determine $r_{1}^{*}$ and $\widetilde{r}_{20}$ in terms of the $\beta$-function coefficients [after eliminating $\bar{r}_{21}$ through eq. (15)]. One finds

$$
\begin{aligned}
r_{1}^{*} & =\beta_{01} \frac{\tilde{\beta}_{20}-\beta_{20}-\left(\beta_{00} / \beta_{01}\right)\left(\tilde{\beta}_{21}-\beta_{21}\right)}{\beta_{11} \beta_{00}-\beta_{01} \beta_{10}} \\
& +\frac{\beta_{00}^{2}}{\beta_{01}} \frac{\tilde{\beta}_{22}-\beta_{22}-\left(\beta_{00} / \beta_{01}\right)\left(\widetilde{\beta}_{23}-\beta_{23}\right)}{\beta_{11} \beta_{00}-\beta_{01} \beta_{10}}
\end{aligned}
$$

with $\tilde{r}_{20}$ following from eq. (17). Eq. (18) is simply the relation derived in ref. [20]:

$r_{\mathbf{1}}^{*}=-\left.\frac{\tilde{\beta_{2}}-\beta_{2}}{\beta_{1}}\right|_{\beta_{0}=0}=-\left.\frac{\tilde{\beta_{2}}-\beta_{2}}{\beta_{1}}\right|_{f=-\beta_{00} / \beta_{01}}$,

which follows more generally from taking the limit $\beta_{0} \rightarrow 0 \quad\left(f=-\beta_{00} / \beta_{01}\right)$ on both sides of eq. (10) and assuming regularity of $r_{1}$ and $r_{2}$ in this limit. Eqs. (14) $-(17)$ show that both $r_{1}^{*}$ and $\bar{r}_{2}$ are determined in terms of $\beta$ - and $\beta_{\text {etr }}$ function coefficients. Note, that requiring $r_{1}=r_{1}^{*}$ does not imply in general that $\bar{r}_{2}$ is $f-$ independent. This can be gained by putting $\bar{r}_{21}=0$, $\bar{r}_{22}=0$ in eqs. (14)-(17). This in turn is equivalent to the unique definition from eqs. (14) and (15) of the part of the NNLO coefficient $\beta_{2}^{*}$ of the $\beta$-function in the prescription considered, namely to

$\beta_{22}^{*}=\widetilde{\beta}_{22}, \quad \beta_{23}^{*}=\widetilde{\beta}_{23}$. 
We are then left with two equations [eqs. (16) and (17) with $\bar{r}_{20}=r_{2}^{*}, \bar{r}_{21}=0, \beta_{21}=\beta_{21}^{*}$ and $\beta_{20}=\beta_{21}^{*}$ ] to determine four unknowns $r_{1}^{*}, r_{2}^{*}, \beta_{21}^{*}$ and $\beta_{20}^{*}$. Even if one adapts the BLM choice of $\mu$ in the MS scheme $\mu^{2}=\mu_{*}^{2}=Q^{2} \operatorname{cxp}\left[-r_{11}^{\overline{M S}}(\mu=Q) / \beta_{01}\right]$, which is equivalent [20] to

$r_{1}^{*}=\left.r_{11}^{\overline{\mathrm{MS}}}\right|_{f=-\beta_{00 / 801}}$,

one does not have cnough equations to determine all unknowns. This simply means that, according to eq. (19), one should also (arbitrarily) fix $\beta_{2}^{*}\left(f=-\beta_{00} /\right.$ $\left.\beta_{01}\right)=\beta_{2}^{\overline{\mathrm{MS}}}\left(f=-\beta_{00} / \beta_{01}\right)$, which thus fixes one linear combination of $\beta_{20}^{*}$ and $\beta_{21}^{*}$ to be equal to its $\overline{\mathrm{MS}}$ value. Note, that eq. (19) shows explicitly [20] that $r_{1}^{*}$ is RS dependent through the $\beta_{2}\left(f=-\beta_{00} / \beta_{01}\right)$ term. This fact can also be seen from eq. (21), if one considers schemes where $r_{1}$ differs from $r_{1}^{\mathrm{MS}}$ by an $f$ independent constant.

One can then further require that both $\beta_{20}^{*}$ and $\beta_{21}^{*}$ be equal to their $\overline{\mathrm{MS}}$ values, however, this looks rather artificial since

$\beta_{22}^{*}=\widetilde{\beta}_{22} \neq \beta_{22}^{\overline{\mathrm{MS}}}, \quad \beta_{23}^{*}=\widetilde{\beta}_{23} \neq \beta_{23}^{\mathrm{M} \mathrm{S}} \equiv 0$,

i.c., one would set a part of $\beta_{2}^{*}$ to be $\beta_{2}^{\overline{M S}}$, and a part of it to be $\widetilde{\beta}_{2}$ ! Further one we will not fix the value of $r_{1}^{*}$ through eq. (21) but consider it as a free quantity.

Similar constraints on the BLM scheme $\beta$-function arise in higher orders. For instance, assuming the arbitrary $f$-independent values $r_{1}^{*}, r_{2}^{*}$ and $r_{3}^{*}$ and the existence in the $\beta_{3} \beta$-functions cocfficients of the $f^{4}$ terms, eq. (11) yields

$\tilde{\beta}_{34}-\beta_{34}^{*}=0, \quad \frac{1}{2}\left(\tilde{\beta}_{33}-\beta_{33}^{*}\right)+\tilde{\beta}_{23} r_{1}^{*}=0$,

$\frac{1}{2}\left(\widetilde{\beta}_{32}-\beta_{32}^{*}\right)+\widetilde{\beta}_{22} r_{1}^{*}=0$,

and

$$
\begin{aligned}
& \beta_{01}\left(r_{3}^{*}-r_{1}^{* 3}\right) \\
& \quad=\frac{5}{2} \beta_{11} r_{1}^{* 2}+\left(3 \widetilde{\beta}_{21}-2 \beta_{21}^{*}\right) r_{1}^{*}+\frac{1}{2}\left(\widetilde{\beta}_{31}-\beta_{31}^{*}\right), \\
& \beta_{00}\left(r_{3}^{*}-r_{1}^{* 3}\right) \\
& \quad=\frac{5}{2} \beta_{10} r_{1}^{* 2}+\left(3 \widetilde{\beta}_{20}-2 \beta_{20}^{*}\right) r_{1}^{*}+\frac{1}{2}\left(\widetilde{\beta}_{30}-\beta_{30}^{*}\right) .
\end{aligned}
$$

Since $\beta_{2}^{*}$ is already fixed in the previous step in terms of $r_{1}^{*}, r_{2}^{*}$, eq. (22) and eqs. (23), (24) clearly determine as expected $\beta_{3}^{*}$ for given $r_{1}^{*}, r_{2}^{*}$ and $r_{3}^{*}$.
Note, that as well as in the NNLO we have more unknowns $\left(\beta_{30}^{*}, \beta_{31}^{*}, \beta_{32}^{*}, \beta_{33}^{*}, \beta_{34}^{*}, r_{3}^{*}\right)$ then available equations (five).

A new feature which appears first at this order is that when $r_{1}^{*}$ is determined in terms of $\beta$-functions by eq. (18) and eqs. (23), (24) this implies one relation between $\beta_{30}^{*}$ and $\beta_{31}^{*}$ (whatever the value of $r_{3}^{*}$ ) which cannot thus be taken as free parameters (contrary to the situation with $\beta_{20}^{*}$ and $\beta_{21}^{*}$ ). In fact, taking the limit $\beta_{0} \rightarrow 0\left(f \rightarrow-\beta_{00} / \beta_{01}\right)$ on both sides of eq. (11), and assuming only regularity of $r_{1}, r_{2}$ and $r_{3}$ in this limit, one easily derives [using eq. (19)] the general relation

$\left.\left(\beta_{1} \beta_{3}-\beta_{2}^{2}\right)\right|_{\beta_{0}=0}=\left.\left(\tilde{\beta}_{1} \tilde{\beta}_{3}-\tilde{\beta}_{2}^{2}\right)\right|_{\beta_{0}=0}$,

i.e., $\beta_{1} \beta_{3}-\beta_{2}^{2}$ is RS invariant when $\beta_{0}=0$ or $f=-\beta_{00} /$ $\beta_{01}$.

Eq. (25) implies the above mentioned relation between $\beta_{30}^{*}$ and $\beta_{31}^{*}$. A more systematic choice of free parameters to all orders, and one which has an analogue in the QED case (see below), might then consist in taking $r_{1}^{*}$ (i.e., $\beta_{2}^{*}$ for $f=-\beta_{00} / \beta_{01}$ ) and $\partial \beta_{i}^{*} / \partial f$ $(i \geqslant 2)$ for $f=-\beta_{00} / \beta_{01}$ as the frec quantities (of course one might also use another set, c.g. $r_{1}^{*}$ and $\left.\beta_{i 1}^{*}\right)$. However, these choices do not help to find a natural physical way to fix them either.

In summary, this first proposed extension of the BLM approach is ambiguous, since it does not allow to fix in a unique way the $f$-independent coefficients $r_{i}^{*}$ for any physical quantity $R$. Given this situation, the simplest and in our opinion most sensible way out remains to take $r_{i}^{*}=0$ (hence $\beta_{i}^{*}=\widetilde{\beta}_{i}$ ) and thus use the effective scheme prescription (note that this is not proposed to be achieved by a mere choice of a renormalization point $\mu$ in a given RS as was incorrectly stated at the end of ref. [1]). The motivations for the effective charge scheme as the RS-invariant method in QCD was argued from more gencral vicwpoints in refs. [5-9].

3. In the above proposed extension of the BLM scheme we tried to adapt the RS to each observable $R(Q)$ in order to obtain $f$-independent coefficients. This required to fix both the scale $\mu$ and the $\beta$-function coefficients $\beta_{i}, i \geqslant 2$ in accordance with a given quantity $R$. Now we will consider a different possi- 
bility, namely to stick to a fixed definition of the coupling (i.e., work with the given $\beta_{i}$, the same for all $R$ ), but to use different scales at each order of perturbation theory, to achieve $f$-independence. We will show that this alternative does not work in general. To see this, let us introduce a set of scales $\mu_{i}$, such that $\mu_{i}$ is fixing the scale of the coupling in the $(i+1)$ st order, and put $\mu_{0}=\lambda_{i} \mu_{i}, i \geqslant 1$.

Let us now use the standard RG relation $a\left(\mu_{0}\right)=$ $a\left(\lambda_{1} \mu_{1}\right)=a\left(\mu_{1}\right)\left[1-\beta_{0} \ln \lambda_{1}^{2} a\left(\mu_{1}\right)+\ldots\right]$ and present the expression for $R$ in the following way:

$$
\begin{aligned}
& R(Q)=a\left(\mu_{0}\right)\left[1+r_{1} a\left(\mu_{0}\right)+r_{2} a^{2}\left(\mu_{0}\right)+\ldots\right] \\
& \quad=a\left(\mu_{0}\right)\left[1+r_{1} a\left(\mu_{1}\right)+\left(r_{2}-r_{1} \beta_{0} \ln \hat{\lambda}_{1}^{2}\right) a^{2}\left(\mu_{1}\right)+\ldots\right] .
\end{aligned}
$$

Choosing now $\mu_{0}=\mu_{*}$ (the BLM prescription), hence $r_{1}=r_{1}^{*}$, it can be shown that this expression and the requirement that $\tilde{r}_{2}=r_{2}^{*}-r_{1}^{*} \beta_{0} \ln \lambda_{1}^{2}$ be $f$-independent give the following set of conditions:

$\widetilde{\beta}_{23}-\beta_{23}=0$

and

$\widetilde{\beta}_{22}-\beta_{22}=\beta_{01}^{2} \ln \dot{\lambda}_{1}^{2} r_{1}^{*}$,

$\beta_{01}\left(\tilde{r}_{2}^{*}-r_{1}^{* 2}\right)=\left(\beta_{11}-2 \beta_{00} \beta_{01} \ln \lambda_{1}^{2}\right) r_{1}^{*}+\widetilde{\beta}_{21}-\beta_{21}$,

$\beta_{00}\left(\tilde{r}_{2}^{*}-r_{1}^{* 2}\right)=\left(\beta_{10}-\beta_{00}^{2} \ln \hat{\lambda}_{1}^{2}\right) r_{1}^{*}+\widetilde{\beta}_{20}-\beta_{20}$.

From eq. (27) one sees that one cannot use a fixed scheme since $\widetilde{\beta}_{23} \neq \beta_{23}$ in general. We conclude this alternative extension of the BLM scheme does not work owing to the eventual presence of $\mathrm{O}\left(f^{3}\right)$-terms in $\widetilde{\beta_{2}}$.

4. We will now show that using the definition of the scale parameter, mentioned in the original BLM paper [1], one can get rid of the $f$-dependent terms in $r_{2}$ starting from the MS-like schemes and thus resolve the ambiguities found in sections 2,3 . Indeed, let us define $\mu^{2}$ in accordance with ref. [1] as

$\mu^{2}=\mu_{0}^{2}\left[1+\eta_{1}(f) a\left(\mu_{0}\right)+\ldots\right]$,

where $\gamma_{1}(f)$ are $f$-dependent functions and $\mu_{0}^{2}$ is the usual scale parameter ${ }^{\# 1}$. Using the explicit dependence of $r_{1}$ upon $Q^{2} / \mu^{2}$, namely

\#I This possibility has been pointed out to us by Brodsky and Hung Jung Lu. For discussions of the general questions, complementary to our analysis, within the framework of the dressed skeleton expansion, see ref. [3].
$r_{1}=r_{1}\left(Q^{2}=\mu^{2}\right)-\beta_{0} \ln \frac{Q^{2}}{\mu^{2}}$,

one can show that the shift $\mu_{0}^{2} \rightarrow \mu^{2}$ of eq. (29) is equivalent to the following redefinition of the coefficients $r_{2}$ :

$r_{2}=r_{2}+\beta_{0} \gamma_{1}(f)$.

Assuming now the polynomial dependence of $\gamma_{1}(f)$,

$\gamma_{1}(f)=\gamma_{10}+\gamma_{11} f$,

we obtain from eq. (10) the following system of linear equations [analogous to those of eqs. (14)-(16) and of eqs. (27)-(28)] which comes from the requirement that $r_{2}^{*}$ must be $f$-independent:

$\tilde{\beta}_{23}-\beta_{23}=\beta_{01}^{2} \gamma_{11}$,

$\widetilde{\beta}_{22}-\beta_{22}=\beta_{01}^{2} \gamma_{10}+2 \beta_{00} \beta_{01} \gamma_{11}$,

$\tilde{\beta}_{21}-\beta_{21}$

$$
=\beta_{01}\left(r_{2}^{*}-r_{1}^{* 2}\right)-\beta_{11} r_{1}^{*}+2 \beta_{00} \beta_{01} \gamma_{10}+\beta_{00}^{2} \gamma_{11},
$$

$\widetilde{\beta}_{20}-\beta_{20}=\beta_{00}\left(r_{2}^{*}-r_{1}^{* 2}\right)-\beta_{10} r_{1}^{*}+\beta_{00}^{2} \gamma_{10}$.

It can be shown that this system is compatible with the relation obtained in eqs. (18), (19) for the NLO coefficient $r_{1}^{*}$ in the BLM prescription.

One can now see that using the definition of the scale parameter of eq. (29) we can absorb the $f^{3}$ coefficient $\widetilde{\beta}_{23}$ of the $\beta_{\text {err }}$ function even within the MSlike schemes (contrarily to the cases discussed in sections 2,3). Indeed, putting $\beta_{23}=\beta \overline{23}=0, \beta_{2 i}=\beta_{2 i}^{\overline{\mathrm{MS}}}$ $(0 \leqslant i \leqslant 2)$, we have four equations [eqs. $(33)-(36)$ ] to determine four unknowns $\left(\gamma_{11}, \gamma_{10}, r_{1}^{*}, r_{2}^{*}\right)$. Therefore, the ambiguities found can be fixed at the NNLO.

5. Let us apply our theoretical considerations to the analysis of the NNLO perturbative QCD approximation of the quantity $R(s)=\sigma_{\mathrm{tot}}\left(\mathrm{e}^{+} \mathrm{e}^{-} \rightarrow\right.$ hadrons $) /$ $\sigma\left(\mathrm{e}^{+} \mathrm{e}^{-} \rightarrow \mu^{+} \boldsymbol{\mu}^{-}\right)$. In the $\overline{\mathrm{MS}}$ scheme the result recently obtained reads [15] (see also ref. [16])

$$
\begin{aligned}
& R(s)=3 \sum Q_{\mathrm{f}}^{2}\left[1+a+(1.986-0.115 f) a^{2}\right. \\
& \left.+\left(-6.637-1.200 f-0.005 f^{2}\right) a^{3}+\ldots\right] \\
& -\left(\sum Q_{\mathrm{f}}\right)^{2} \cdot 1.239 a^{3},
\end{aligned}
$$


where $Q_{\mathrm{f}}$ are the quark charges and $a=a\left(\mu_{\mathrm{MS}}^{2}=s\right)$. Using now the results of calculations of the coefficients of the $\beta$-function in the MS-like schemes at the three-loop level [18], reminded now in the numerical form

$\beta_{0}=2.75-0.167 f, \quad \beta_{1}=6.375-0.792 f$,

$\beta_{2}=22.320-4.370 f+0.094 f^{2}$,

we obtain the numerical expression of the schemeinvariant $\widetilde{\beta}_{2}$ without taking into account proportional to $\left(\sum Q_{\mathrm{f}}\right)^{2}$ light-by-light-type contribution to $R(s)$ :

$\widetilde{\beta_{2}}=-19.422-2.334 f+0.076 f^{2}+0.003 f^{3}$

(compare with the similar expression obtained in ref. [15]). Fixing now the NNLO ambiguities of the BLM approach in accordance with the discussions of section 4 , we get the following numerical values of the scale fixing parameters introduced in eqs. (30), (31):

$\mu_{0}^{2}=\mu_{\mathrm{BLM}}^{2}=\mu \frac{2}{\mathrm{MS}} \exp (0.69)$,

$\gamma_{11}=0.11, \quad \gamma_{10} \approx 3$

and the NNLO approximation of $R(s)$ in the considered generalization of the BLM approach:

$$
\begin{aligned}
& R(s)=3 \sum Q_{\mathrm{f}}^{2}\left(1+a_{*}+0.08 a_{*}^{2}-23.3 a_{*}^{3}\right) \\
& -\left(\sum Q_{\mathrm{f}}\right)^{2} \cdot 1.24 a_{*}^{3},
\end{aligned}
$$

where $a_{*}=a\left\{\mu_{0}^{2}\left[1+\gamma_{1}(f) a\left(\mu_{0}^{2}\right)\right]=s\right\}$.

6. Let us now discuss the QED case. The corresponding results can be obtained from the $\mathrm{QCD}$ ones after putting $C_{\mathrm{A}}=0, C_{\mathrm{F}}=1$ and $T f=N$ where $C_{\mathrm{A}}$ and $C_{\mathrm{F}}$ are the corresponding Casimir operators, $T=\frac{1}{2}$ and $N$ is the number of lepton types. As a result, in QED $\beta_{i 0} \equiv 0$, which represents the main difference for the present discussion with respect to the QCD case. Since $\beta_{i 0} \equiv 0$, there is no analogue in QED of the relation of eq. (19), so that $r_{i}^{*}$ is not fixed in terms of $\beta$-function coefficients. Otherwise, the main conclusions remain similar: one can find RS with $n$-independent coefficients $r_{i}^{*}$ for a given $R(Q)$ either by assigning arbitrary values to the $r_{i}^{*}$ themselves, or, alternatively consider $r_{i}^{*}$ and the coefficients $\beta_{i 1}^{*}$ of the terms linear in $N$ in the $\beta$-function coefficients as the free parameters, as is clear from eqs. (14) $-(16)$ with $\beta_{00}=0$.

We note that in general the $\beta_{i 1}$-coefficient $(i \geqslant 0)$ are not RS invariant, even in QED: the known fact that in QED $\beta_{i 1}^{\mathrm{MS}}=\beta_{i 1}^{\mathrm{MOM}}=\beta_{i 1}^{\mathrm{OS}}$ for the MS-like, the momentum (MOM) and the on-shell (OS) subtraction schemes is a consequence of the absence of an $\mathrm{O}\left(N^{0}\right)$-term in the expression coefficients $r_{i}$ for the standard QED invariant charge $\alpha_{\text {inv }}\left(Q^{2}\right)=$ $\alpha_{\text {MOM }}\left(Q^{2}\right)=\alpha /(1+\alpha \Pi)=\alpha\left(1+r_{1} \alpha+\ldots\right)$, where $\alpha$ corresponds to the renormalized coupling constant of the MS-like or OS schemes, so that $\lim r_{i}(N \rightarrow 0)=0$, and $\alpha_{\mathrm{inv}} \equiv \alpha_{\mathrm{MOM}}$ coincides with $\alpha_{\overline{\mathrm{MS}}}$ (or $\alpha_{\mathrm{MS}}$ ) and $\alpha_{\text {os }}$ in the limit $N \rightarrow 0$. This statement follows immediately from the QED analogue of eq. (10) [or eq. (35) ], which gives in the limit $N \rightarrow 0$ (or $\beta_{00}=0$ )

$r_{20}=r_{10}^{2}+\frac{\beta_{11}}{\beta_{01}} r_{10}+\frac{\tilde{\beta}_{21}-\beta_{21}}{\beta_{01}}$,

so that $r_{10}=r_{20}=0$ clearly implies $\widetilde{\beta}_{21}=\beta_{21}$. In gencral, however, $r_{10}$ and $r_{20}$ do not vanish (note that $r_{10}$ is just the BLM coefficient $r_{1}^{*}$ in QED!) and consequently $\widetilde{\beta}_{21} \neq \beta_{21}$. This has been explicitly checked to be the case for the QED part of the NNLO QCD result [15] for $R(s)$ (sce also ref. [16]). The result $r_{10}=r_{1} \quad(N=0) \neq 0$ also holds in the case of the $(g-2)_{\mu}$ quantity, calculated in the OS scheme at the four-loop lcvel [22] and discussed in ref. [1].

Taking now the frec parameters to be $r_{10}$ and the $\beta_{10}^{*}$, onc can choose $\beta_{i 1}^{*}=\beta_{i 1}^{\mathrm{MS}} \equiv \beta_{i 1}^{\text {oS }}$. Then, only $r_{10}$ remains free and sensible choices are $r_{10}=r_{1}^{*}$ (which will insure the corresponding RS coupling $\alpha_{*}$ to coincide with $\alpha_{\mathrm{MS}}, \alpha_{\mathrm{OS}}$ and $\alpha_{\mathrm{inv}}$ as $N \rightarrow 0$ ) or even $r_{1}^{*}=$ 0 . Note that $r_{1}^{*}=0$ does not in general imply $r_{i}^{*}=0$ $(i \geqslant 2)$ if $\beta_{i 1}^{*}=\beta_{i 1}^{\mathrm{MS}} \neq \widetilde{\beta}_{i 1}$, i.c., we will not recover in this case the effective charge prescription (which is equivalent, under the assumption of $N$-independent $r_{i}$, to $r_{i}^{*}=0$ and $\left.\beta_{i 1}^{*}=\widetilde{\beta}_{i 1}\right)$. However, given the other relations like $\beta_{23}^{*}=\widetilde{\beta}_{23}$ and $\beta_{22}^{*}=\widetilde{\beta}_{22}$ (which are necessary for $N$-independence within the framework of considerations of section 2) the most natural choice for this purpose in this case remains the effective charges prescription.

However, as in QCD one can consider the alternative higher order extension of the BLM scheme which uses a coupling constant dependent definition of the scale via eq. (29) (sec ref. [1]). In this case it is possible to achieve $N$-independence at the NNLO by fixing $\gamma_{1}(f)$-function coefficients through eqs. (33), (34) with $\beta_{00}=0, \beta_{23}=0$ [it should be stressed that, as well as in the QCD case, the corresponding coefficients of the $\beta_{\text {eir }}(R)$-functions, namely $\widetilde{\beta}_{2}$ contain the 
additional $N^{3}$ terms $\left.\widetilde{\beta}_{23}\right]$ and $\beta_{22}=\beta_{22}^{\mathrm{MS}}$ or $\beta_{22}=\beta_{22}^{\text {OS }}$. Therefore, as well as in QCD, one can realize the BLM idcas starting from the initial $\overline{\mathrm{MS}}$ or OS schemes. The concrete QED examples will be considered elsewhere.

7. Finally, we remark that the question of reliability of perturbative QCD is a priori distinct from the problem of fixing the ambiguities in the BLM approach in higher orders. We emphasize that in the framework of the effective charges scheme the applicability of perturbative QCD to a given physical quantity requires only the applicability of perturbation theory to the RS invariant equations (6), (7). This in turn depends solely on the valuc of the ratio $Q^{2} / \Lambda_{\text {eff }}^{2}$ and on the perturbative behaviour of the $\beta_{\text {eff }}(R)$-function, which has no straightforward relation to the valuc of $r_{1}^{*}$ (except in the QCD case at $f=-\beta_{00} / \beta_{01}$ through the rclation of eq. (20) derived in ref. [20]). If the expansion of the $\beta_{\mathrm{eff}}(R)$-function for some possible observed values of $R$ happens to converge poorly (this sometimes happens for not so large values of $\alpha_{\mathrm{s}}$, sec in particular the discussions of the characteristics of the NNLO approximations for $\mathrm{H}^{0} \rightarrow$ hadrons $[14,13]$ and $\mathrm{e}^{+} \mathrm{e}^{-} \rightarrow$ hadrons [15]) the transformation of the results from the effective charges scheme to the BLM-type scheme where $r_{i}^{*} \neq$ 0 will simply transfer the bad convergence properties in the lower region of energies from the $\beta_{\mathrm{cm}}(R)$-scries to the $R\left(a^{*}\right)$-series (compare eq. (39) and eq. (41), see also the independent discussions of similar topics in ref. [8] and ref. [13]). Moreover, even if the NLO BLM condition indicates large values of $r_{1}^{*}$ one can use a variant of the effective charge scheme, proposed in ref. [23].

We are grateful to our colleagues from the Centre de Physique Théorique, Ecolc Polytechnique, where the work has been started, for discussions. One of us (A.K.) wishes to thank the members of the Randall Laboratory of Physics, University of Michigan, for hospitality in Ann Arbor where the preliminary version of this work [24] has been written and G. Lepage and P. Mackenzic for the definite comments. G.G. is grateful to his collcagues from the Thcory Division of the Institute for Nuclear Rescarch for hospitality in Moscow where this work was completed. Special thanks go to S. Brodsky and Hung Jung Lu for the interest in the work and valuable suggestions which resulted in the extensive revision of the work [24] and in the foundation of the prescription discussed in sections 4, 5 to extend the BLM approach to the NNLO of perturbative QCD.

\section{References}

[1] S.J. Brodsky, G.P. Lepage and P.B. Mackenzie, Phys. Rev. D 28 (1983) 228.

[2] DELPHI Collab., P.Abreu et al., Phys. Lett. B 252 (1990) 149;

MARK II Collab., S. Komammiya et al., Phys. Rev. Lett. 64 (1990) 987.

[3] Hung Jung Lu, SLAC preprint SLAC-PUB-5577 (1991), submitted to Phys. Rev. D;

Hung Jung Lu and C.A.R. Sá de Melo, Phys. Lett. B 273 (1991) 260.

[4] W. Bardeen, A. Buras, D. Duke and T. Muta, Phys. Rev. D 18 (1978) 3998.

[5] G. Grunberg, Phys. Lett. B 95 (1980) 70; B 110 (1982) 501 (E).

[6] G. Grunberg, Ecole Polytechnique preprint A510.0782 (1982), unpublished.

[7] G. Grunberg, Phys. Rev. D 29 (1984) 2315.

[8] G. Grunberg, Phys. Rev. D 40 (1989) 680.

[9] A.L. Kataev, N.V. Krasnikov and A.A. Pivovarov, Nucl. Phys. B 198 (1982) 508

[10] P.M. Stevenson, Phys. Rcv. D 23 (1981) 2916.

[11] A. Dhar, Phys. Lett., B 128 (1983) 407; A. Dhar and V. Gupta, Phys. Rev. D 29 (1984) 2822.

[12] P.M. Stevenson, Phys. Rev. D 33 (1986) 3130.

[13] A.L. Kataev, Talk QCD-90 Conf. (Montpellier, July 1990), in: Proc. QCD-90 Conf., ed. S. Narison, Nucl. Phys. B (Proc. Suppl.) 23B (1991) 72.

[14] S.G. Gorishny, A.L. Kataev, S.A. Larin and L.R. Surguladze, Phys. Rev. D 43 (1991) 1633.

[15] S.G. Gorishny, A.L. Kataev and S.A. Larin, Standard model and bcyond, in: Proc. first Intern. Workshop CERN-IHEPJINR (October 1990), cds. S. Dubnicka, D. Ebert and A. Sazonov (World Scientific, Singapore); Phys. Lett. B 259 (1991) 144.

[16] L.R. Surguladze and M.A. Samuel, Phys. Rev. Lett. 66 (1991) 560; Phys. Rev. Lett. 66 (1991) 2416 (E).

[17] A.L. Katacv, University of Michigan preprint UM-TH-9104 (1991), unpublished.

[18] O.V. Tarasov, A.A. Vladimirov and A.Yu. Zharkov, Phys. Lett. B 93 (1980) 429.

[19] D.V. Shirkov and O.V. Tarasov, JINR Rapid Commun. 3 (29) (1988) 18;

O.V. Tarasov and D.V. Shirkov, Yad. Fiz. 51 (1990) 1389.

[20] G. Grunberg, Phys. Lett. B 135 (1984) 455

[21] W. Celmaster and P.M. Stevenson, Phys. Lett. B 125 (1983) 493

[22] T. Kinoshita, B. Nizic and Y. Okamoto, Phys. Rev. D 41 (1990) 593.

[23] G. Grunberg, Ecole Polytechnique preprint A063.0791 (1991).

[24] G. Grunberg and A.L. Kataev, University of Michigan preprint UM-TH-91-(07 (1991). 